\title{
Dark Matter “Collider” from Inelastic Boosted Dark Matter
}

\author{
Doojin Kim, ${ }^{1,2}$ Jong-Chul Park, ${ }^{3, *}$ and Seodong Shin ${ }^{4,5,6,+}$ \\ ${ }^{1}$ Theory Department, CERN, CH-1211 Geneva 23, Switzerland \\ ${ }^{2}$ Department of Physics, University of Florida, Gainesville, Florida 32611, USA \\ ${ }^{3}$ Department of Physics, Chungnam National University, Daejeon 34134, Republic of Korea \\ ${ }^{4}$ Department of Physics and IPAP, Yonsei University, Seoul 03722, Republic of Korea \\ ${ }^{5}$ Enrico Fermi Institute, University of Chicago, Chicago, Illinois 60637, USA \\ ${ }^{6}$ Physics Department, Indiana University, Bloomington, Indiana 47405, USA
}

(Received 5 January 2017; revised manuscript received 28 August 2017; published 18 October 2017)

\begin{abstract}
We propose a novel dark matter (DM) detection strategy for models with a nonminimal dark sector. The main ingredients in the underlying DM scenario are a boosted DM particle and a heavier dark sector state. The relativistic DM impinged on target material scatters off inelastically to the heavier state, which subsequently decays into DM along with lighter states including visible (standard model) particles. The expected signal event, therefore, accompanies a visible signature by the secondary cascade process associated with a recoiling of the target particle, differing from the typical neutrino signal not involving the secondary signature. We then discuss various kinematic features followed by DM detection prospects at large-volume neutrino detectors with a model framework where a dark gauge boson is the mediator between the standard model particles and DM.
\end{abstract}

DOI: 10.1103/PhysRevLett.119.161801

Introduction.-The direct detection of dark matter (DM) is a major strategy for probing its nongravitational interactions with standard model (SM) particles. Various experiments have been proposed and performed over the past decades, mostly focusing on nonrelativistic, elastic scattering signatures in a detector medium. Most of them, however, have set strong constraints to DM models rather than discovered unambiguous signatures. This situation has motivated alternative detection schemes.

One class of strategies is to look for nonrelativistic but inelastic scattering signals. Relevant DM models typically imagine that a DM particle scatters off to an excited state (if kinematically allowed) in which the target-recoil spectrum differs from that in the elastic scattering [1]. The excited state can be deexcited back to the DM state, potentially accompanying SM states such as $\mathrm{x}$ rays, which may leave observable signatures inside a detector [2]. In both cases, an observation of both a target recoiling and secondary visible particle(s) often suffers from DM kinetic energy insufficient to overcome the relevant thresholds simultaneously.

Another category of alternatives is to search for relativistic scattering signatures of DM. While dominant (thermal) relic DM is nonrelativistic, boosted DM may be produced in the laboratory or in the context of extended dark-sector scenarios. An example mechanism for the former is the

Published by the American Physical Society under the terms of the Creative Commons Attribution 4.0 International license. Further distribution of this work must maintain attribution to the author(s) and the published article's title, journal citation, and DOI. decay of an energetic mediator created via high-intensity (proton or electron) beam bombardment on fixed target material [3-9]. A representative example for the latter is the so-called boosted dark matter (BDM) framework [10-13], in which there exist two DM components whose stability is ensured by separate unbroken symmetries. In typical models, the lighter species directly annihilates to SM particles, whereas the heavier one is in indirect contact with thermal bath through the lighter species; i.e., the only possible direct annihilation channel of the heavier DM pair is into a lighter DM pair. Their respective relic is determined by the assisted freeze-out mechanism [14], often rendering the heavier (lighter) dominant (subdominant). In terrestrial experiments, the signature expected under the BDM framework is relativistic, elastic scattering of lighter $\mathrm{DM}$, as the lighter becomes boosted in the present universe due to the mass gap between the two DM species. We remark that the scattering of energetic lighter DM with target material would resemble the neutrino scattering via neutral current interactions. Moreover, the typical flux of such lighter DM is as small as $O\left(10^{-7} \mathrm{~cm}^{-2} \mathrm{~s}^{-1}\right)$ for the heavier DM mass of $O(10 \mathrm{GeV})$; thus, large-volume neutrino detectors are preferred to observe such energetic and rare signals [10-13]. While resulting phenomenology is intriguing, we point out that it is hard to discern a relativistic scattering signal of DM from a neutrino scattering event, the main irreducible background.

We emphasize that an extended dark sector structure may allow us to circumvent challenges existing in previous search schemes. In this sense, we propose a novel channel in which additional (unstable) dark sector particles play a 


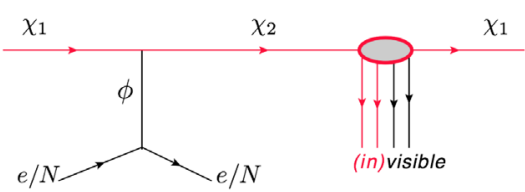

FIG. 1. Inelastic boosted DM direct-detection scenarios under consideration.

key role in search for the relativistic DM scattering signals arising in various models comprising them. Essential features are schematically depicted in Fig. 1, where the scattered dark sector particle (denoted by $\chi_{2}$ ) differs from the incoming DM (denoted by $\chi_{1}$ ); i.e., an inelastic scattering occurs in the recoil of the target. Furthermore, $\chi_{2}$ is heavier than $\chi_{1}$, so that the former subsequently decays into lighter states including the latter and visible SM particles, which is reminiscent of typical cascade decay signatures in collider experiments [15]. The expected signal, therefore, involves a recoiling of target material and (visible) decay products from the secondary process of $\chi_{2}$. We first find that this feature is clearly distinctive from highly energetic neutrino signatures in DM detection.

Model framework.-To validate the DM scenario explained above, we first delineate a DM model framework which contains the cascade process depicted in Fig. 1. Employing a Dirac fermionic DM $\chi_{1}$ for simplicity, we assume that it interacts with target SM particles (e.g., electron or nucleus) via a $t$-channel exchange of the mediator $\phi$. As stated earlier, we further assume that the outgoing dark sector particle is not $\chi_{1}$ but a heavier unstable particle $\chi_{2}$ (i.e., $m_{\chi_{2}}>m_{\chi_{1}}$ ). In principle, the mediator $\phi$ can be either a SM or a new physics particle, but we take a "dark" gauge boson $X_{\mu}$ for simplicity from the following toy model Lagrangian with a dark $\mathrm{U}(1)_{\mathrm{X}}$ gauge symmetry:

$$
\mathcal{L}_{X} \supset-\frac{\sin \epsilon}{2} F_{\mu \nu} X^{\mu \nu}+g_{12} \bar{\chi}_{2} \gamma^{\mu} \chi_{1} X_{\mu}+\text { H.c. }
$$

where the first term describes the kinetic mixing between $\mathrm{U}(1)_{\mathrm{X}}$ and $\mathrm{U}(1)_{\mathrm{EM}}$ [17-24] parameterized by $\epsilon$. The offdiagonal gauge interaction of $\chi_{1}$ and $\chi_{2}$ with $X_{\mu}$ appears in the second term with coupling $g_{12}$. We expect that such a vertex may arise, e.g., from the mixing in the dark sector after imposing different $\mathrm{U}(1)_{\mathrm{X}}$ charges to $\chi_{1}$ and $\chi_{2}$ [see also Ref. [25] for the mixing in the SM quark sector with a $\mathrm{U}(1)^{\prime}$ symmetry]. More concrete model building including other possible scenarios (e.g., Higgs portal) will be available in our future work [26].

The heavier nature of $\chi_{2}$ renders its decay eventually into $\chi_{1}$ plus SM or other (invisible) dark sector particles. Such a decay, in general, proceeds via a sequential cascade process as symbolized by a red-circled blob in Fig. 1. Hence, the expected signal event is featured by a recoil of the target SM particle, accompanying secondary signatures from the cascade decay process. As a minimal choice, we take a single-step cascade decay of $\chi_{2}$ throughout this Letter; i.e., $\chi_{2}$ decays back into $\chi_{1}$ and $\phi$ by the interactions in Eq. (1).

We first calculate the matrix element squared for the process $\chi_{1} T \rightarrow \chi_{2} T$ with $T$ being the associated target,

$$
\begin{aligned}
\overline{|\mathcal{M}|^{2}=} & \frac{8\left(\epsilon e g_{12}\right)^{2} m_{T}}{\left[2 m_{T}\left(E_{\chi_{2}}-E_{\chi_{1}}\right)-m_{\phi}^{2}\right]^{2}} \\
& \times\left\{\mathcal{M}_{0}\left(F_{1}+\kappa F_{2}\right)^{2}+\mathcal{M}_{1}\left[-\left(F_{1}+\kappa F_{2}\right) \kappa F_{2}\right.\right. \\
& \left.\left.+\left(\kappa F_{2}\right)^{2}\left(E_{\chi_{1}}-E_{\chi_{2}}+2 m_{T}\right) /\left(4 m_{T}\right)\right]\right\} .
\end{aligned}
$$

Here $\mathcal{M}_{0}$ and $\mathcal{M}_{1}$ are defined as follows:

$$
\begin{aligned}
\mathcal{M}_{0}= & {\left[m_{T}\left(E_{\chi_{1}}^{2}+E_{\chi_{2}}^{2}\right)-\left(\delta m_{\chi}\right)^{2}\left(E_{\chi_{2}}-E_{\chi_{1}}+m_{T}\right) / 2\right.} \\
& \left.+m_{T}^{2}\left(E_{\chi_{2}}-E_{\chi_{1}}\right)+m_{\chi_{1}}^{2} E_{\chi_{2}}-m_{\chi_{2}}^{2} E_{\chi_{1}}\right], \\
\mathcal{M}_{1}= & m_{T}\left\{\left[\left(E_{\chi_{1}}+E_{\chi_{2}}\right)-\left(m_{\chi_{2}}^{2}-m_{\chi_{1}}^{2}\right) /\left(2 m_{T}\right)\right]^{2}\right. \\
& \left.+\left(E_{\chi_{1}}-E_{\chi_{2}}+2 m_{T}\right)\left[\left(E_{\chi_{2}}-E_{\chi_{1}}\right)-(\delta m)^{2} /\left(2 m_{T}\right)\right]\right\},
\end{aligned}
$$

where $\delta m_{\chi} \equiv m_{\chi_{2}}-m_{\chi_{1}}$ and $E_{\chi_{1(2)}}$ is the $\chi_{1(2)}$ energy measured in the laboratory frame [27]. For the two form factors $F_{1}$ and $F_{2}$, we set them to be 1 and 0 for the electron target (or $e$ scattering), whereas we employ nontrivial values as per Ref. [28] for the proton target (or $p$ scattering) together with the proton anomalous magnetic moment $\kappa=1.79$.

Kinematic features.-We now discuss interesting kinematic features arising in the model framework discussed earlier. Like ordinary colliders, the maximum mass reach of $\chi_{2}$ is $\sqrt{s}-m_{T}$ with $\sqrt{s}$ being the overall center-of-mass energy (i.e., $s=m_{T}^{2}+2 E_{\chi_{1}} m_{T}+m_{\chi_{1}}^{2}$ ),

$$
m_{\chi_{2}} \leq \sqrt{m_{T}^{2}+2 E_{\chi_{1}} m_{T}+m_{\chi_{1}}^{2}}-m_{T} \text {. }
$$

If $\chi_{1}$ is much heavier than the target (i.e., $m_{\chi_{1}} \gg m_{T}$ ) along with a decent boost $\gamma_{\chi_{1}}$ [29], the above relation is approximated to

$$
m_{\chi_{2}} \lesssim m_{\chi_{1}}+\left(\gamma_{\chi_{1}}-1\right) m_{T},
$$

to which our $e$ scattering corresponds. On the other hand, the opposite limit, $m_{\chi_{1}} \ll m_{T}$, results in

$$
m_{\chi_{2}} \lesssim \gamma_{\chi_{1}} m_{\chi_{1}}
$$

allowing us to probe much heavier dark sector states than the incoming DM, which is possible for $p$ scattering.

We next discuss the expected energy spectra of the recoiling target and the visible particles from the secondary vertex. In the laboratory frame, the differential cross section is

$$
\frac{d \sigma}{d E_{T}}=\frac{m_{T}}{8 \pi \lambda\left(s, m_{T}^{2}, m_{\chi_{1}}^{2}\right)} \overline{|\mathcal{M}|^{2}},
$$


where $E_{T}$ is the total energy of the recoiling target and $\lambda(x, y, z)=(x-y-z)^{2}-4 y z$. Here $\left.\overline{|\mathcal{M}|}\right|^{2}$ is expressed in terms of $E_{T}=E_{\chi_{1}}+m_{T}-E_{\chi_{2}}$. We find that kinematically allowed maximum (minimum) total energy of the recoiling target $E_{T}^{+}\left(E_{T}^{-}\right)$is

$E_{T}^{ \pm}=\frac{s+m_{T}^{2}-m_{\chi_{2}}^{2}}{2 \sqrt{s}} \frac{E_{\chi_{1}}+m_{T}}{\sqrt{s}} \pm \frac{\lambda^{1 / 2}\left(s, m_{T}^{2}, m_{\chi_{2}}^{2}\right)}{2 \sqrt{s}} \frac{p_{\chi_{1}}}{\sqrt{s}}$

where $p_{\chi_{1}}=\sqrt{E_{\chi_{1}}^{2}-m_{\chi_{1}}^{2}}$. Note that the measured energy in neutrino experiments corresponds to the magnitude of the spatial momentum (equivalently, the kinetic energy) of the recoiling target, so we will use $p_{T}=\sqrt{E_{T}^{2}-m_{T}^{2}}$ as target recoil energy (distinguished from corresponding total energy) unless otherwise specified. The upper-left panel ( $e$ scattering) and the lower-left panel ( $p$ scattering) in Fig. 2 demonstrate expected unit-normalized recoil energy (i.e., $p_{T}$ ) spectra for our four reference points ( $e$-ref. 1, $e$-ref. 2, $p$-ref. 1, and $p$-ref. 2) as detailed in the plots, which are not only safe from various experimental bounds [30,31] but phenomenologically well motivated [3-9,9,20,21,32,33]. Note that the differential cross section is greater for the smaller momentum transfer as expected in Eq. (2).

The spectral behavior in the distribution of total energy of $\phi\left(E_{\phi}\right)$, in principle, depends on the relevant vertex structure. In our toy model, due to the vectorlike nature of the mediator coupling, $\chi_{2}$ is produced in an unpolarized way, so that it can be treated effectively as a scalar. For a simple two-body decay, the energy spectra of decay products have been extensively examined in the context of collider phenomenology [34-40] and cosmic-ray phenomenology [41-45]. For generality, we consider the case that $m_{\phi}$ is not negligible, finding the following expression based on the formulation in Ref. [39]:
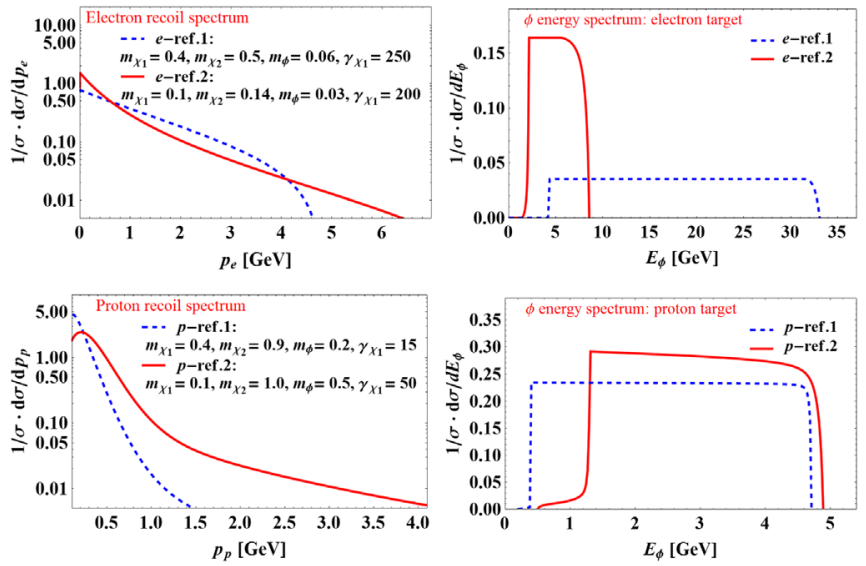

FIG. 2. Expected unit-normalized energy spectra of the recoiling target particles from the primary vertex (left panels) and outgoing mediators from the secondary vertex (right panels) for $e$ scattering (top panels) and $p$ scattering (bottom panels). The reference masses are in unit of $\mathrm{GeV}$.

$$
\frac{d \sigma}{d E_{\phi}}=\int d \gamma_{\chi_{2}} \frac{d \sigma}{d \gamma_{\chi_{2}}} \frac{1}{2 E_{\phi}^{*} \sqrt{\gamma_{\chi_{2}}^{2}-1}},
$$

where $E_{\phi}^{*}$ is the $\phi$ energy measured in the $\chi_{2}$ rest frame. The detailed expressions for the integral range are not illustrative, so we instead refer to Refs. [26,39]. Here the boost distribution of $\chi_{2}, d \sigma / d \gamma_{\chi_{2}}$, can be easily obtained from the $\chi_{2}$ energy spectrum, which is, in turn, derived from Eq. (8) with $E_{T}$ replaced by $E_{\chi_{1}}+m_{T}-E_{\chi_{2}}$.

The expected (unit-normalized) energy spectra of $\phi$ produced in the cascade process for our reference points are exhibited in the upper-right panel ( $e$ scattering) and the lower-right panel ( $p$ scattering) in Fig. 2, respectively. For the chosen reference points, we find that $E_{\chi_{2}}$ values are highly localized towards the kinematic endpoint; therefore, the resulting $\phi$ energy spectrum appears almost boxlike. In practice, the energy of $\phi$ can be measured from its visible decay products. Throughout this Letter, we assume that the mediator $\phi$ predominantly decays into $e^{+} e^{-}$[5]. We then find the decay $\chi_{2} \rightarrow \chi_{1} \phi \rightarrow \chi_{1} e^{+} e^{-}$occurs within at most $\lesssim 1 \mathrm{~cm}$ for our reference points, which is below the detector resolutions identifying separate vertices [e.g., a few $\mathrm{cm}$ for the Deep Underground Neutrino Experiment (DUNE) [46]]. Depending on $m_{\phi}$ and $\epsilon$, one may also consider an appreciable displaced vertex in the decay $\chi_{2} \rightarrow \chi_{1} e^{+} e^{-}$ which can be observed at detectors with high vertexposition resolution [e.g., DUNE and Search for Hidden Particles (SHiP)] [26]. In either case, a signal event is characterized by a recoiling target ( $e$ or $p$ ) and an $e^{+} e^{-}$ pair, so the angular separations among them would be critical to identify the signal events; this will be discussed in the next section.

Detection prospects.-Based on the signal features discussed so far, we are now in the position to assess the detection prospects of our signal. In order for our signal to be sensitive even with small flux, we choose large volume neutrino detectors: Super-Kamiokande (SK), Hyper-Kamiokande (HK), and DUNE, where the latter two are future proposals. We summarize their key attributes in Table I. While we do our analysis having in mind a BDM-type scenario for obtaining boosted DM, we again emphasize that fixed target experiments [e.g., DUNE at Long-Baseline Neutrino Facility (LBNF) [46], SHiP [50], and T2HKK [51]] are alternative sources $[8,9,26]$.

TABLE I. Summary of the volume, threshold (kinetic) energy, and angular resolution of considered experiments from Refs. [47-49] for SK, HK, and DUNE, respectively. $p_{e}^{\text {th }}$ at SK and $\mathrm{HK}$ could be lowered below $0.1 \mathrm{GeV}$ with worse angular resolution. Angular resolution gets better with higher $p_{T}$.

\begin{tabular}{lccccc}
\hline \hline Experiment & Volume $(\mathrm{Mt})$ & $p_{e}^{\text {th }}(\mathrm{GeV})$ & $p_{p}^{\text {th }}(\mathrm{GeV})$ & $\theta_{e}^{\text {res }}$ & $\theta_{p}^{\text {res }}$ \\
\hline SK & 0.0224 & 0.1 & 1.07 & $3^{\circ}$ & $3^{\circ}$ \\
HK & 0.56 & 0.1 & 1.07 & $3^{\circ}$ & $3^{\circ}$ \\
DUNE & 0.04 & 0.03 & 0.05 & $1^{\circ}$ & $5^{\circ}$ \\
\hline \hline
\end{tabular}



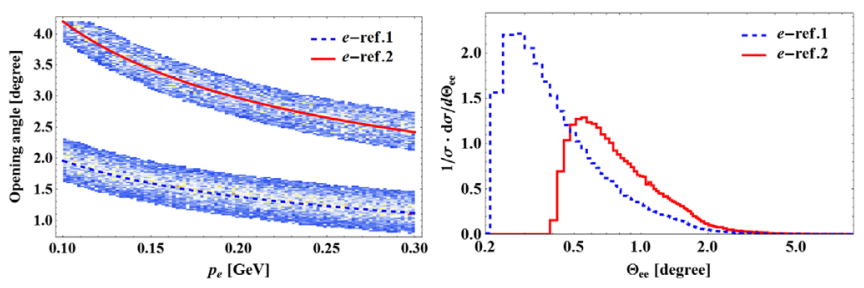

FIG. 3. Left: Angular separation between the recoiling target and $\chi_{2}$ (red solid and blue dashed lines) or the mediator $\phi$ (temperature-scaled regions). Right: Angular separation between the $e^{+} e^{-}$pair from the $\phi$ decay.

The energy and angular resolutions for $e$ scattering are usually better than those for $p$ scattering, especially in SK and HK. This is because large momentum transfer above $m_{p}=$ $0.938 \mathrm{GeV}$ is required for the recoiling proton to produce Cherenkov radiation ( $p_{p} \gtrsim 1.07 \mathrm{GeV}$ for SK and HK [47]). Note that this requirement is rather relaxed in the liquid $\mathrm{Ar}$ time projection chamber detectors of DUNE. Recalling the trend that the differential cross section is larger for smaller momentum transfer, one can expect that $e$ scattering is preferred over $p$ scattering in SK and HK if focusing only on the recoil signal. For $p$ scattering, we further restrict ourselves to $p_{p} \lesssim 1.8 \mathrm{GeV}$ to avoid the possibility of deep inelastic scattering [52].

As stated before, observation of the secondary cascade signal plays the key role in discovery of our DM signal. We point out that the visible particles are often collimated due to the large boost of the incident DM. Therefore, unambiguous signal identification depends on what extent we can separate those (highly) collimated signals beyond the angular resolutions of the detectors. Defining $\theta_{\chi_{2}}$ as the angle between the recoiling target and $\chi_{2}$ in the laboratory frame, we obtain

$$
\cos \theta_{\chi_{2}}=\frac{E_{T} E_{\chi_{2}}+\left(m_{T}^{2}+m_{\chi_{2}}^{2}-s\right) / 2}{\sqrt{\left(E_{\chi_{2}}^{2}-m_{\chi_{2}}^{2}\right)\left(E_{T}^{2}-m_{T}^{2}\right)}} .
$$

The value $\theta_{\chi_{2}}$ roughly determines the angular separation between the primary and secondary signals when $\chi_{2}, \phi$, and the decay products $\left(e^{+} e^{-}\right)$are highly collimated. This is true for $e$ scattering, as shown in the left panel of Fig. 3. The red solid and blue dashed lines (temperature-scaled bands) show the angle between the recoiling target and $\chi_{2}(\phi)$, from which we clearly see that $\chi_{2}$ and $\phi$ are collimated. We further check that the angular separation between $e^{+}$and $e^{-}$from the $\phi$ decay are mostly within $1.5^{\circ}$, as shown in the right panel of Fig. 3. Adopting the angular resolution $3^{\circ}$ for SK and HK, we find that our reference point $e$-ref. 2 manifests two separable signatures for most momentum values of the recoiling electron $p_{e} \in[0.1,0.3] \mathrm{GeV}$ (see also Table I) [53].

On the other hand, larger angular separation is possible for our $p$-scattering reference points because $E_{\chi_{1}}, m_{p}$, and $m_{\chi_{2}}$ are roughly of the same order, so that typical $\chi_{2} \mathrm{~s}$ are neither too boosted nor too aligned along the recoiling proton direction. In addition, we observe that the opening angle of $\phi$ decay products (defined as $\Theta_{e e}$ ) gets larger. Given a mediator boost factor $\gamma_{\phi}$, we find

$$
\Theta_{e e} \simeq \arccos \left[1-2 /\left(\gamma_{\phi}^{2} \sin ^{2} \theta+\cos ^{2} \theta\right)\right],
$$

where $\theta$ is the emission angle of one of the decay products with respect to the $\phi$ boost direction in the $\phi$ rest frame. Here we took the fact that $m_{\phi} \gg m_{e}$ for all our reference points. It is easy to see that the opening angle is greater than $6^{\circ}$ for all possible $\theta$ as far as $\gamma_{\phi} \lesssim 20$. We then find that our reference points selected for $p$ scattering are anticipated to have three resolvable signatures in most of the allowed phase space, whereas those for $e$ scattering would involve two signatures. This is an unarguable advantage of $p$ scattering, although the cross section is smaller than that for $e$ scattering.

In both the $e$-scattering and $p$-scattering cases, we expect to observe two or three separate signatures, which are not expected in usual neutrino scattering. Thus, it is fair to obtain the experimental sensitivity by requiring three signal events which correspond to the $95 \%$ C.L. upper limit under the assumption of a null observation over a null background with Poisson statistics (see also Refs. [54,55] for related discussion), while we leave more systematic background analysis to future work. We list the minimum required fluxes of $\chi_{1}$ making our reference points sensitive in SK, HK, and DUNE in Table II. Considering the fact that the typical flux of $\chi_{1}$ demanded in the minimal BDM setup is $O\left(10^{-7}\right) \mathrm{cm}^{-2} \mathrm{~s}^{-1}$ [10], we see that $e$-ref. 2 is rather promising. The other reference points can be also probed once we consider a modified BDM setup to increase the flux up to $O\left(10^{-4}\right) \mathrm{cm}^{-2} \mathrm{~s}^{-1}[12,13]$ or fixed-target experiments with much higher intensity [7-9,26]. Note that the sensitivities in HK 1-year data is much better, compared to SK 13.6-year data, mainly due to the bigger volume. For $p$

TABLE II. Required fluxes with which our reference points become sensitive in various experiments.

\begin{tabular}{lccccc}
\hline \hline Experiment & $\begin{array}{c}\text { Run } \\
\text { time }(\mathrm{yr})\end{array}$ & $\begin{array}{c}e \text {-ref. 1 } \\
\left(10^{-7} \mathrm{~cm}^{-2} \mathrm{~s}^{-1}\right)\end{array}$ & $\begin{array}{c}e \text {-ref. 2 } \\
\left(10^{-7} \mathrm{~cm}^{-2} \mathrm{~s}^{-1}\right)\end{array}$ & $\begin{array}{c}p \text {-ref. 1 } \\
\left(10^{-7} \mathrm{~cm}^{-2} \mathrm{~s}^{-1}\right)\end{array}$ & $\begin{array}{c}p \text {-ref. 2 } \\
\left(10^{-7} \mathrm{~cm}^{-2} \mathrm{~s}^{-1}\right)\end{array}$ \\
\hline SK & 13.6 & 170 & 7.1 & 3500 & 5200 \\
HK & 1 & 88 & 3.7 & 1900 & 2800 \\
HK & 13.6 & 6.7 & 0.28 & 140 & 210 \\
DUNE & 1 & 190 & 9.0 & 150 & 1600 \\
DUNE & 13.6 & 14 & 0.69 & 11 & 120 \\
\hline \hline
\end{tabular}



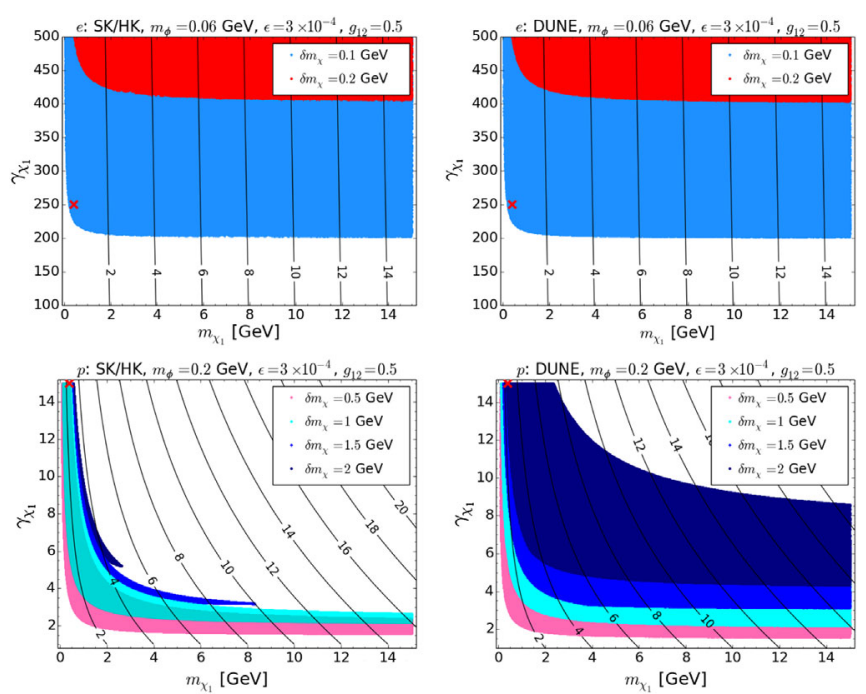

FIG. 4. $e$ scattering (upper panels) and $p$ scattering (lower panels) parameters in SK and HK (left panels) and DUNE (right panels). For $e$ scattering we scan for $\delta m_{\chi}=0.1,0.2 \mathrm{GeV}$ while for $p$ scattering we scan for $\delta m_{\chi}=0.5,1,1.5,2 \mathrm{GeV}$. Black contours show maximally allowed $m_{\chi_{2}}$ values in $\mathrm{GeV}$ and the red crosses show our reference parameters.

scattering we observe that the sensitivities increase in DUNE due to its remarkably lower $p_{p}^{\text {th }}$.

Finally, we conduct a parameter scan to check the viability of our signal processes in a wider range of space. Fixing $m_{\phi}=0.06(0.2) \mathrm{GeV}, \epsilon=3 \times 10^{-4}$, and $g_{12}=0.5$, we obtain the allowed parameter region [56] of $e(p)$ scattering in the $m_{\chi_{1}}$ vs $\gamma_{\chi_{1}}$ plane for $\delta m_{\chi}=0.1,0.2 \mathrm{GeV}$ $(0.5,1,1.5,2 \mathrm{GeV})$ and show them in the upper (lower) panels of Fig. 4. The left (right) panels are for SK and HK (DUNE). The black contours represent the maximally accessible $m_{\chi_{2}}$ for a given set of $m_{\chi_{1}}$ and $\gamma_{\chi_{1}}$ [see Eq. (5) as well]. The minimally required $\chi_{1}$ fluxes for our signal to be sensitive in each experiment are an order of magnitude smaller than (of the same order as) those for $e$ scattering ( $p$ scattering) in Table II. The red $X$ points in Fig. 4 denote the reference points: $e$-ref. 1 and $p$-ref. 1 .

Future prospect.-Similar analyses will be straightforwardly applicable to future fixed-target experiments. It is possible to study more complicated signatures such as multistep cascade decays, although we employed the simplest secondary process in this Letter. Furthermore, we expect proactive utilization of the knowledge from collider phenomenology due to the similarity of the proposed DM scenario with typical collider signatures, when detectors are designed and implemented accordingly in the future. As a concluding remark, we strongly encourage DM-related intensity-frontier collaborations (e.g., DUNE at LBNF, SHiP, and T2HKK) to pay attention to the proposal in this Letter as possible physics to pursue.

We thank K. Agashe, M. Aoki, J.-H. Huh, D. Kim, K. Kong, K. Matchev, T. Saab, C. S. Shin, M. Son, and J. Yoo for insightful and useful discussions. We appreciate Brookhaven Forum for its encouraging environment enabling us to initiate this project, and CETUP* (Center for Theoretical Underground Physics and Related Areas), Santa Fe LHC summer workshop, and Focus Workshop on Particle Physics and Cosmology by Institute for Basic Science-Center for Theoretical Physics of the Universe (IBS-CTPU) for their hospitality during the completion of this work. D. K. was supported in part by DOE Grant No. DE-SC0010296, and is presently supported by the Korean Research Foundation (KRF) through the CERNKorea Fellowship program. J.C. P. is supported by the National Research Foundation of Korea (Grant No. NRF2016R1C1B2015225) and the POSCO Science Fellowship of the POSCO TJ Park Foundation. S. S. was supported in part by DOE Grant No. DE-SC0010120 and the National Research Foundation of Korea (Grant No. NRF2017R1D1A1B03032076).

*jcpark@cnu.ac.kr shinseod@indiana.edu

[1] D. Smith and N. Weiner, Phys. Rev. D 64, 043502 (2001).

[2] M. Pospelov, N. Weiner, and I. Yavin, Phys. Rev. D 89, 055008 (2014).

[3] B. Batell, M. Pospelov, and A. Ritz, Phys. Rev. D 80, 095024 (2009).

[4] P. deNiverville, M. Pospelov, and A. Ritz, Phys. Rev. D 84, 075020 (2011).

[5] E. Izaguirre, G. Krnjaic, P. Schuster, and N. Toro, Phys. Rev. D 90, 014052 (2014).

[6] M. Battaglieri et al. (BDX Collaboration), arXiv:1607.01390.

[7] B. A. Dobrescu and C. Frugiuele, J. High Energy Phys. 02 (2015) 019.

[8] P. Coloma, B. A. Dobrescu, C. Frugiuele, and R. Harnik, J. High Energy Phys. 04 (2016) 047.

[9] P. deNiverville, C. Y. Chen, M. Pospelov, and A. Ritz, Phys. Rev. D 95, 035006 (2017).

[10] K. Agashe, Y. Cui, L. Necib, and J. Thaler, J. Cosmol. Astropart. Phys. 10 (2014) 062.

[11] J. Berger, Y. Cui, and Y. Zhao, J. Cosmol. Astropart. Phys. 02 (2015) 005.

[12] K. Kong, G. Mohlabeng, and J. C. Park, Phys. Lett. B 743, 256 (2015).

[13] H. Alhazmi, K. Kong, G. Mohlabeng, and J. C. Park, J. High Energy Phys. 04 (2017) 158.

[14] G. Belanger and J. C. Park, J. Cosmol. Astropart. Phys. 03 (2012) 038.

[15] A similar cascade process can be used in the context of explaining cosmic-ray excesses [16].

[16] D. Kim, J. C. Park, and S. Shin, arXiv:1702.02944.

[17] L. B. Okun, Zh. Eksp. Teor. Fiz. 83, 892 (1982) [Sov. Phys. JETP 56, 502 (1982)].

[18] P. Galison and A. Manohar, Phys. Lett. 136B, 279 (1984).

[19] B. Holdom, Phys. Lett. 166B, 196 (1986). 
[20] J. H. Huh, J. E. Kim, J. C. Park, and S. C. Park, Phys. Rev. D 77, 123503 (2008).

[21] M. Pospelov, A. Ritz, and M. B. Voloshin, Phys. Lett. B 662, 53 (2008).

[22] E. J. Chun, J. C. Park, and S. Scopel, J. High Energy Phys. 02 (2011) 100.

[23] J. C. Park and S. C. Park, Phys. Lett. B 718, 1401 (2013).

[24] G. Blanger, A. Goudelis, J. C. Park, and A. Pukhov, J. Cosmol. Astropart. Phys. 02 (2014) 020.

[25] J. E. Kim, M. S. Seo, and S. Shin, Phys. Rev. D 83, 036003 (2011).

[26] D. Kim, J. C. Park, and S. Shin (to be published).

[27] Obviously, for the fixed-target experiments, the target frame is the same as the laboratory frame.

[28] I. A. Qattan et al., Phys. Rev. Lett. 94, 142301 (2005).

[29] Note that $\gamma_{\chi_{1}}$ is the boost factor of $\chi_{1}$, i.e., $E_{\chi_{1}}=\gamma_{\chi_{1}} m_{\chi_{1}}$.

[30] R. Essig et al., arXiv:1311.0029.

[31] D. Banerjee et al. (NA64 Collaboration), Phys. Rev. Lett. 118, 011802 (2017).

[32] N. Arkani-Hamed, D. P. Finkbeiner, T. R. Slatyer, and N. Weiner, Phys. Rev. D 79, 015014 (2009).

[33] E. J. Chun and J. C. Park, J. Cosmol. Astropart. Phys. 02 (2009) 026.

[34] K. Agashe, R. Franceschini, and D. Kim, Phys. Rev. D 88, 057701 (2013).

[35] K. Agashe, R. Franceschini, D. Kim, and K. Wardlow, Phys. Dark Universe 2, 72 (2013).

[36] K. Agashe, R. Franceschini, and D. Kim, J. High Energy Phys. 11 (2014) 059.

[37] C. Y. Chen, H. Davoudiasl, and D. Kim, Phys. Rev. D 89, 096007 (2014).

[38] K. Agashe, R. Franceschini, D. Kim, and K. Wardlow, J. High Energy Phys. 05 (2016) 138.

[39] K. Agashe, R. Franceschini, S. Hong, and D. Kim, J. High Energy Phys. 04 (2016) 151.

[40] K. Agashe, R. Franceschini, D. Kim, and M. Schulze, Eur. Phys. J. C 76, 636 (2016).
[41] F.W. Stecker, Cosmic Gamma Rays, NASA Special Publication Technical Report No. 249 (NASA, Washington, 1971), https://ntrs.nasa.gov/search.jsp?R=19710015288.

[42] D. Kim and J. C. Park, Phys. Dark Universe 11, 74 (2016).

[43] D. Kim and J. C. Park, Phys. Lett. B 750, 552 (2015).

[44] K. K. Boddy, K. R. Dienes, D. Kim, J. Kumar, J. C. Park, and B. Thomas, Phys. Rev. D 94, 095027 (2016).

[45] K. K. Boddy, K. R. Dienes, D. Kim, J. Kumar, J. C. Park, and B. Thomas, Phys. Rev. D 95, 055024 (2017).

[46] R. Acciarri et al. (DUNE Collaboration), arXiv:1601.02984.

[47] M. Fechner et al. (Super-Kamiokande Collaboration), Phys. Rev. D 79, 112010 (2009).

[48] E. Kearns et al. (Hyper-Kamiokande Working Group Collaboration), arXiv:1309.0184.

[49] R. Acciarri et al. (DUNE Collaboration), arXiv:1512.06148.

[50] M. Anelli et al. (SHiP Collaboration), arXiv:1504.04956.

[51] K. Abe et al. (Hyper-Kamiokande Proto-Collaboration), arXiv:1611.06118.

[52] Of course, one could perform a dedicated analysis for deep inelastic scattering signatures, although we do not do so here. In this sense, our results for the $p$ scattering can be considered conservative.

[53] For $e$-ref. 1, detecting events with separable signatures is very challenging according to the left panel of Fig. 3; this forces us to perform a more careful analysis with regard to angular separation by considering both the recoil electron and the $e^{+} e^{-}$pair from the $\phi$ decay.

[54] R. Dermisek, E. Lunghi, and S. Shin, J. High Energy Phys. 10 (2016) 081.

[55] R. Dermisek, J. P. Hall, E. Lunghi, and S. Shin, J. High Energy Phys. 12 (2014) 013.

[56] For $p$ scattering, we define the allowed parameter region to satisfy $\sigma_{\text {R.O.I }} / \sigma_{\text {tot }}>0.5$, where $\sigma_{\text {tot }}$ is the total cross section in the whole kinematically allowed range of $\left|\vec{p}_{p}\right|$ and $\sigma_{\text {R.O.I }}$ is the region of interest in which the momentum transfer to the target proton is not too large to break up the proton (practically, $\left|\vec{p}_{p}\right| \in\left[p_{p}^{\text {th }}, 1.8 \mathrm{GeV}\right]$ ). 